\title{
THE DIVERGENCE BEHAVIOR OF ADAPTIVE SIGNAL PROCESSING ALGORITHMS WITH FINITE SEARCH HORIZON
}

\author{
Holger Boche, Volker Pohl \\ Lehrstuhl für Theoretische Informationstechnik \\ Technische Universität München, 80333 München, Germany \\ \{boche, volker.pohl\}@tum.de
}

\begin{abstract}
Many important non-adaptive approximation methods are know to diverge for almost all functions from certain Banach space $\mathcal{X}$. One can show that a corresponding adaptive method will improve this behavior in the sense that it converges to the desired result for almost all functions in $\mathcal{X}$. However, even though an adaptive method tries to find an optimal approximation for any given function, the search horizon (i.e. the search set) has to be finite in practical applications.

This paper shows that an adaptive method with finite search horizon either converges for all $f \in \mathcal{X}$ or it diverges for almost all $f \in \mathcal{X}$. As an example, we show that there exists no realizable adaptive method which can calculate the Hilbert transform of a continuous function $f$ based on samples of $f$.
\end{abstract}

Index Terms - Adaptive signal processing, Hilbert transform, Sampled data

\section{INTRODUCTION \& MOTIVATION}

Let $\mathcal{X}, \mathcal{Y}$ be Banach spaces and let $\mathrm{T}: \mathcal{X} \rightarrow \mathcal{Y}$ be a bounded linear operator. An important problem in many applications of signal processing is to approximate $\mathrm{T}$ by a sequence $\left\{\mathrm{T}_{N}\right\}_{N \in \mathbb{N}}$ of linear, bounded operators $\mathrm{T}_{N}: \mathcal{X} \rightarrow \mathcal{Y}$. The operators $\mathrm{T}_{N}$ often have a particular structure which is determined by the actual application, or they are chosen to make the evaluation of $\mathrm{T}_{N} f$ simple. For example, in digital signal processing, one naturally requires that the calculation of $\mathrm{T}_{N} f$ is based on a finite number $\left\{f\left(\lambda_{n, N}\right)\right\}_{n=1}^{N}$ of samples of $f$. Then the operators $\mathrm{T}_{N}$ will have the form $\mathrm{T}_{N} f=$ $\sum_{n=1}^{N} f\left(\lambda_{n, N}\right) p_{n, N}$ where $p_{n} \in \mathcal{X}$ are kernels which are often simple in some sense. In many cases $p_{n, N}$ are chosen to be exponential, trigonometric, or spline functions [1], since they allow for a very fast and efficient calculation. Usually, it is not hard to design sequences $\left\{\mathrm{T}_{N}\right\}_{N \in \mathbb{N}}$ such that $\mathrm{T}_{N} f$ converges to $\mathrm{T} f$ for all $f$ in a dense subset $\mathcal{X}_{0}$ of $\mathcal{X}$. The fundamental question is then whether $\left\{\mathrm{T}_{N} f\right\}_{N \in \mathbb{N}}$ converges to $\mathrm{T} f$ for every $f \in \mathcal{X}$.

There are many important problems where $\mathrm{T}_{N} f$ actually fails to converge for all $f \in \mathcal{X}$. Examples include the Fourier series on the space $\mathcal{C}(-\pi, \pi)$ of continuous functions, the Shannon sampling series on the Paley-Wiener space $\mathcal{P} \mathcal{W}^{1}$ of integrable bandlimited functions, or Hilbert transform approximations on $\mathcal{C}(-\pi, \pi)$ [2]. Such negative outcomes are often stated as

$$
\limsup _{N \rightarrow \infty}\left\|\mathrm{T}_{N} f_{*}-\mathrm{T} f_{*}\right\|_{\mathcal{Y}}=\infty \quad \text { for some } f_{*} \in \mathcal{X} .
$$

Results of this form are typically proven by showing that the norms $\left\|\mathrm{T}_{N}\right\|_{\mathcal{X} \rightarrow \mathcal{Y}}$ are not uniformly bounded. Then (1) follows from the

This work was partly supported by the German Research Foundation (DFG) under Grant BO 1734/20-1 and PO 1347/2-1. theorem of Banach-Steinhaus [3]. Moreover, the Banach-Steinhaus technique implies additionally that the set of all $f_{*} \in \mathcal{X}$ which satisfy (1) is a residual set $[4,5]$, i.e. a large set in $\mathcal{X}$ (see Sec. 2 for a precise description).

Non-adaptive and adaptive approximations The sequence $\left\{\mathrm{T}_{N}\right\}_{N \in \mathbb{N}}$ can be regarded as an approximation method for $\mathrm{T} f$. It is non-adaptive, since the sequence $\left\{\mathrm{T}_{N}\right\}_{N \in \mathbb{N}}$ does not depend on the actual $f \in \mathcal{X}$. A result as in (1) tells us that the non-adaptive method is actually not applicable since it diverges for most $f \in \mathcal{X}$.

However, (1) shows only that the sequence $\left\{\mathrm{T}_{N}\right\}_{N \in \mathbb{N}}$ has "bad subsequences", indexed by $\left\{N_{k}\right\}_{k \in \mathbb{N}}$, such that $\mathrm{T}_{N_{k}} f_{*}$ does not converge to $\mathrm{T} f_{*}$. However, (1) does not exclude the existence of "good subsequences" such that $\left\{\mathrm{T}_{N_{k}} f_{*}\right\}_{k \in \mathbb{N}}$ converges to $\mathrm{T} f_{*}$. More precisely, (1) does not exclude

$\liminf _{N \rightarrow \infty}\left\|\mathrm{T}_{N} f-\mathrm{T} f\right\|_{\mathcal{Y}}<\infty$ or even $\liminf _{N \rightarrow \infty}\left\|\mathrm{T}_{N} f-\mathrm{T} f\right\|_{\mathcal{Y}}=0$

for all $f \in \mathcal{X}$. If a convergent subsequence $\left\{N_{k}\right\}_{k \in \mathbb{N}} \subset \mathbb{N}$ exists, it generally depends on the actual $f \in \mathcal{X}$ [6]. So the selection of a good subsequence $\left\{N_{k}=N_{k}(f)\right\}_{k \in \mathbb{N}}$ such that

$$
\lim _{k \rightarrow \infty}\left\|\mathrm{T}_{N_{k}(f)} f-\mathrm{T} f\right\|_{\mathcal{Y}}=0
$$

can be regarded as an adaption of the approximation method $\left\{\mathrm{T}_{N}\right\}_{N \in \mathbb{N}}$ to the actual function $f \in \mathcal{X}$. Conversely, it is clear that no convergent subsequence exists, if in addition to (1)

$$
\liminf _{N \rightarrow \infty}\left\|\mathrm{T}_{N} f_{*}-T f_{*}\right\|_{\mathcal{Y}}=\infty \quad \text { for some } f_{*} \in \mathcal{X},
$$

a property which was coined strong divergence of $\left\{\mathrm{T}_{N}\right\}_{N \in \mathbb{N}}$ [7]. The next example illustrates that there are sequences $\left\{\mathrm{T}_{N}\right\}_{N \in \mathbb{N}}$ which diverge as in (1) but which are not strongly divergent.

Example 1 (Fourier Series): Let $\mathcal{X}=\mathcal{C}(\mathbb{T})$ be the set of all continuous functions $f$ on $\mathbb{T}=[-\pi, \pi]$ with $f(-\pi)=f(\pi)$, equipped with the maximum norm $\|\cdot\|_{\infty}$. Let $\mathcal{Y}=\mathbb{C}$ and consider for an arbitrary but fixed $\lambda \in \mathbb{T}$ the bounded linear functional $\mathrm{T}_{\lambda} f=f(\lambda)$. To define an approximation sequence $\left\{\mathrm{T}_{\lambda, N}\right\}_{N \in \mathbb{N}}$ of $\mathrm{T}_{\lambda}$, we consider for any $f \in \mathcal{C}(\mathbb{T})$ and all $N \in \mathbb{N}$ the partial Fourier series

$$
\left(\mathrm{F}_{N} f\right)(t)=\sum_{n=-N}^{N} \widehat{f}_{n} \mathrm{e}^{\mathrm{i} n t} \quad \text { with } \quad \widehat{f}_{n}=\frac{1}{2 \pi} \int_{\mathbb{T}} f(\tau) \mathrm{e}^{-\mathrm{i} n \tau} \mathrm{d} \tau
$$

and define $\mathrm{T}_{\lambda, N}: \mathcal{C}(\mathbb{T}) \rightarrow \mathbb{C}$ by $\mathrm{T}_{N, \lambda} f=\left(\mathrm{F}_{N} f\right)(\lambda)$.

It is known that $\left\|\mathrm{T}_{\lambda, N}\right\|=\left\|\mathrm{F}_{N}\right\|_{\mathcal{C}(\mathbb{T}) \rightarrow \mathcal{C}(\mathbb{T})} \rightarrow \infty$ as $N \rightarrow \infty$ [8]. So by the theorem of Banach-Steinhaus, $\left\{\mathrm{T}_{\lambda, N}\right\}_{n \in \mathbb{N}}$ has a divergence behavior as in (1). However, a result of Fejér [9] implies that 


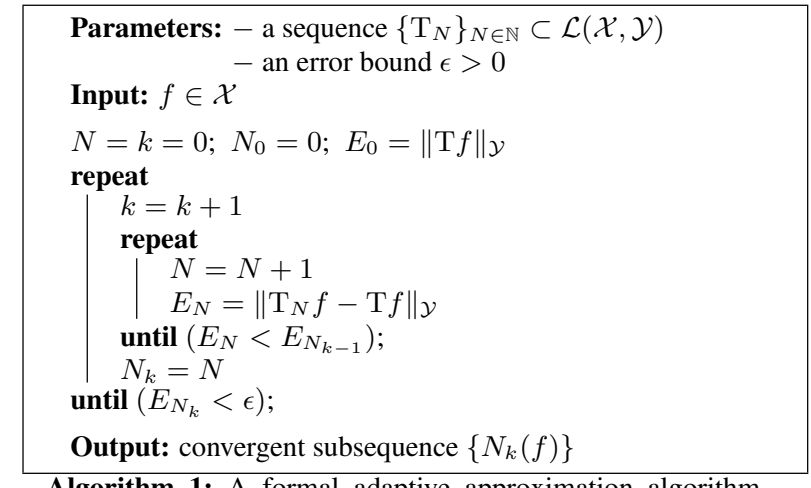

Algorithm 1: A formal adaptive approximation algorithm with infinite search horizon.

to every $f \in \mathcal{C}(\mathbb{T})$ there is a subsequence $\left\{N_{k}=N_{k}(\lambda, f)\right\}_{k \in \mathbb{N}}$ so that

$$
\lim _{k \rightarrow \infty} \mathrm{T}_{\lambda, N_{k}} f=\mathrm{T}_{\lambda} f=f(\lambda)
$$

A similar same results holds for system approximations [10].

\section{PROBLEM STATEMENT AND NOTATIONS}

Given a sequence $\left\{\mathrm{T}_{N}\right\}_{N \in \mathbb{N}}$ which satisfies (1). Let

$$
\mathcal{W}_{\mathrm{T}}=\left\{f \in \mathcal{X}: \liminf _{N \rightarrow \infty}\left\|\mathrm{T}_{N} f-\mathrm{T} f\right\|_{\mathcal{Y}}=0\right\}
$$

be the set of all $f \in \mathcal{X}$ for which a subsequence $\left\{N_{k}\right\}_{k \in \mathbb{Z}}$ exists such that $\mathrm{T}_{N_{k}} f$ converges to $\mathrm{T} f$. This paper studies the following two questions:

- Is it possible to find practical (i.e. realizable) adaptive algorithms which determines for a given $f \in \mathcal{W}_{\mathrm{T}}$ the convergent subsequence $\left\{N_{k}(f)\right\}_{k \in \mathbb{N}}$ such that (2) is satisfied?

- Which size has the subset of functions in $\mathcal{W}_{\mathrm{T}}$ for which such a practical algorithm fails?

We recall shortly (see, e.g., $[5,11]$ ) that a set $\mathcal{M}$ in a topological space $\mathcal{X}$ is called nowhere dense if its closure does not contain any nonempty open set of $\mathcal{X}$. A set $\mathcal{M}$ is said to be meager (or of first category) if $\mathcal{M}$ is the countable union of nowhere dense sets. A set which is not meager, is called nonmeager (or of second category). Meager sets are "small". They play a similar role for topological spaces as sets of measure zero in measure spaces [11]. The complement of any meager set is called a residual set. It follows from Baire's theorem that any residual set is nonmeager and dense. Moreover, any open and dense subset is a residual set.

The set of all linear bounded operators $\mathrm{T}: \mathcal{X} \rightarrow \mathcal{Y}$ is denoted by $\mathcal{L}(\mathcal{X}, \mathcal{Y})$, and $\mathcal{L}(\mathcal{X})$ stands for $\mathcal{L}(\mathcal{X}, \mathcal{X})$. Throughout this paper we consider the following class of operator sequences.

Definition 1: A sequence $\left\{\mathrm{T}_{N}\right\}_{N \in \mathbb{N}} \subset \mathcal{L}(\mathcal{X}, \mathcal{Y})$ is said to be a weakly divergent approximation sequence of $\mathrm{T} \in \mathcal{L}(\mathcal{X}, \mathcal{Y})$ if

(i) there is a dense subset $\mathcal{X}_{0}$ of $\mathcal{X}$ such that

$$
\lim _{N \rightarrow \infty}\left\|\mathrm{T}_{N} f-\mathrm{T} f\right\|_{\mathcal{Y}}=0 \quad \text { for all } f \in \mathcal{X}_{0} .
$$

(ii) it is weakly divergent, i.e. it satisfies (1).

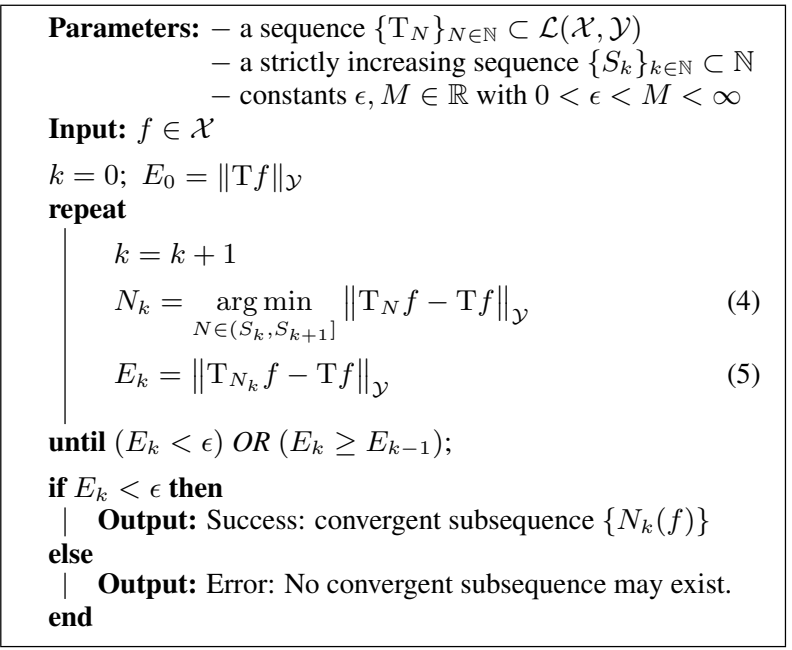

Algorithm 2: A formal adaptive algorithm with finite search horizons $\left(S_{k}, S_{k-1}\right]$.

\section{ADAPTIVE TECHNIQUES}

Methods with infinite search horizon Given a weakly divergent approximation sequence of $\mathrm{T} \in \mathcal{L}(\mathcal{X}, \mathcal{Y})$. It was shown in [10] that the corresponding set $\mathcal{W}_{\mathrm{T}}$ is a residual set in $\mathcal{X}$. So for almost every function $f \in \mathcal{X}$ it is possible to find a subsequence $\left\{N_{k}=\right.$ $\left.N_{k}(f)\right\}_{k \in \mathbb{N}}$ such that $\lim _{k \rightarrow \infty}\left\|\mathrm{T}_{N_{k}} f-\mathrm{T} f\right\|_{\mathcal{Y}} \rightarrow 0$. This result shows the power of adaptive approximation methods, because (1) tells us that the non-adaptive method $\left\{\mathrm{T}_{N}\right\}_{N \in \mathbb{N}}$ fails to converge for almost all functions in $\mathcal{X}$ whereas an adapted method $\left\{\mathrm{T}_{N_{k}(f)}\right\}_{k \in \mathbb{N}}$ succeeds for almost all $f \in \mathcal{X}$.

The determination of a convergent subsequence has generally to be done by an exhaustive search as sketched in Algorithm 1. To this end, we calculate the approximation errors $E_{N}=\left\|\mathrm{T}_{N} f-\mathrm{T} f\right\|_{\mathcal{Y}}$ for $N=1,2,3, \ldots$ and extract a strictly decreasing subsequence $\left\{E_{N_{k}}\right\}$. The algorithm terminates if $E_{N_{k}}$ falls below a certain given bound $\epsilon>0$. As a result, for any given $f \in \mathcal{W}_{\mathrm{T}}$ one obtains the desired convergent subsequence $\left\{N_{k}(f)\right\}$.

However, it should be noted that the algorithm may not terminate, namely if $f \notin \mathcal{W}_{\mathrm{T}}$, i.e. if $f$ satisfies (3). A second difficulty of Algorithm 1 is that the gap between two consecutive indices $N_{k}$ and $N_{k+1}$ can be arbitrary large. Then Algorithm 1 may run practically a very long time until it finds the correct subsequence. Since the length of the search intervals are not bounded in this algorithm, we speak of an algorithm with infinite search horizon in each step.

Methods with finite search horizon To overcome the described problems of Algorithm 1, one introduces a criterion which guarantees that the search algorithm terminates after a reasonable time, even if the error criterion is not satisfied. A practical realization of such a procedure is described in Algorithm 2. The behavior of the algorithm is determined by a predefined strictly increasing sequence $\left\{S_{k}\right\}_{k \in \mathbb{N}} \subset \mathbb{N}$. For $k=1,2, \ldots$, the algorithm observes the approximation error $\left\|\mathrm{T}_{N} f-\mathrm{T} f\right\| \mathcal{Y}$ for all $N$ in the $k$-th interval $\left(S_{k}, S_{k+1}\right]$ and chooses the optimal index $N_{k}$ according to (4). If the approximation error (5) in step $k$ is smaller than in preceding step $k-1$, we proceed in the same way with $k+1$ until the approximation error falls below a desired bound $\epsilon$. Otherwise, if in the 
interval $\left(S_{k}, S_{k+1}\right]$ no improvement can be achieved, the algorithm stops with an error, assuming that no convergent subsequence exists.

The sequence $\left\{S_{k}\right\}_{k \in \mathbb{N}}$ determines the search horizon in every step $k$. It is equal to the length of the interval $\left(S_{k}, S_{k+1}\right]$. For example, we may choose $S_{k}=k \alpha$ with a certain $\alpha \in \mathbb{N}$. Then the search horizon is equal to $\alpha$ for every $k \in \mathbb{N}$. Alternatively, we may choose $S_{k}=\alpha^{k}$. Then the search horizon increases exponentially with $k$. In any case, the search horizon is always finite and the algorithm terminates if it finds no improvement of the approximation error on the predefined search intervals.

In contrast to Algorithm 1, Algorithm 2 may not be able to find a convergent subsequence for all $f \in \mathcal{W}_{\mathrm{T}}$ since it may terminate after the predefine number of steps although there exists a convergent subsequence. However, we may expect that if the search intervals $\left(S_{k}, S_{k+1}\right]$ are sufficiently large then Algorithm 2 will fail only for very few functions in $\mathcal{W}_{\mathrm{T}}$.

Formal problem formulation The interesting question is now, whether this intuition is correct and how large we have to choose the search intervals $\left(S_{k}, S_{k+1}\right]$ such that Algorithm 2 terminates successfully for almost every $f \in \mathcal{W}_{\mathrm{T}}$.

Formally, we investigate the following related problem.

Problem 1: Let $\left\{\mathrm{T}_{N}\right\}_{N \in \mathbb{N}}$ be a weakly divergent approximation sequence of $\mathrm{T} \in \mathcal{L}(\mathcal{X}, \mathcal{Y})$. Does there exist a strictly increasing sequence $\left\{S_{k}\right\}_{k \in \mathbb{N}} \subset \mathbb{N}$ such that for every $f \in \mathcal{W}_{\mathrm{T}}$ there is a subsequence $\left\{N_{k}(f)\right\}_{k \in \mathbb{N}}$ such that for all $k \in \mathbb{N}$ we have

$$
N_{k} \in\left(S_{k}, S_{k+1}\right] \text { and } \sup _{k \in \mathbb{N}}\left\|\mathrm{T}_{N_{k}(f)} f-\mathrm{T} f\right\|_{\mathcal{Y}}<\infty .
$$

Remark: Note that Problem 1 only requires that the approximation error remains bounded. We actually do not require that the error goes to zero. So it is clear that if Problem 1 has no solution, then there exist functions $f \in \mathcal{W}_{\mathrm{T}}$ such that Algorithm 2 fails in finding a convergent subsequence $\left\{N_{k}(f)\right\}_{k \in \mathbb{N}}$ such that (2) holds.

The following lemma can be used to check whether Problem 1 is solvable or not. It is clear that Problem 1 has no solution if to every strictly increasing sequence $\left\{S_{k}\right\}_{k \in \mathbb{N}} \subset \mathbb{N}$ there is an $f \in \mathcal{X}$ such that for all sequences $\left\{N_{k}\right\}_{k \in \mathbb{N}}$ with $N_{k} \in\left(S_{k}, S_{k+1}\right]$ always

$$
\limsup _{k \rightarrow \infty}\left\|\mathrm{T}_{N_{k}} f-\mathrm{T} f\right\|_{\mathcal{Y}}=\infty \text {. }
$$

More precisely, we have the following statement.

Lemma 1: Problem 1 has no solution if and only if to every strictly increasing sequence $\left\{S_{k}\right\}_{k \in \mathbb{N}} \subset \mathbb{N}$ there exists an $f \in \mathcal{X}$ such that

$$
\limsup _{k \rightarrow \infty}\left(\min _{N \in\left(S_{k}, S_{k+1}\right]}\left\|\mathrm{T}_{N} f-\mathrm{T} f\right\|_{\mathcal{Y}}\right)=\infty .
$$

Proof: It is obvious that Problem 1 is not solvable if (7) holds. Conversely, assume Problem 1 is not solvable and (7) is not satisfied. We show hat this yields a contradiction. If (7) does not hold, then there exists a strictly increasing sequence $\left\{\widetilde{S}_{k}\right\}_{k \in \mathbb{N}}$ such that

$$
\limsup _{k \rightarrow \infty} \min _{N \in\left(\widetilde{S}_{k}, \widetilde{S}_{k+1}\right]}\left\|\mathrm{T}_{N} f-\mathrm{T} f\right\|_{\mathcal{Y}}<\infty \quad \text { for all } f \in \mathcal{X} .
$$

So for any $f \in \mathcal{X}$ there is a sequence $\left\{N_{k}=N_{k}(f)\right\}_{k \in \mathbb{N}}$ with $N_{k} \in\left(\widetilde{S}_{k}, \widetilde{S}_{k+1}\right]$ and such that for all $k \in \mathbb{N}$

$$
\left\|\mathrm{T}_{N_{k}} f-\mathrm{T} f\right\|_{\mathcal{Y}}=\min _{N \in\left(\widetilde{S}_{k}, \widetilde{S}_{k+1}\right]}\left\|\mathrm{T}_{N} f-\mathrm{T} f\right\|_{\mathcal{Y}}<\infty .
$$

Thus $\sup _{k \in \mathbb{N}}\left\|\mathrm{T}_{N_{k}} f-\mathrm{T} f\right\|_{\mathcal{Y}}<\infty$. Since $\left\{N_{k}(f)\right\}_{k \in \mathbb{N}}$ is strictly increasing we solved Problem 1 and arrived at a contradiction.

\section{THE SIZE OF THE DIVERGENCE SETS}

Let $\left\{\mathrm{T}_{N}\right\}_{N \in \mathbb{N}}$ be a weakly divergent approximation sequence of $\mathrm{T} \in \mathcal{L}(\mathcal{X}, \mathcal{Y})$. As we discussed in Section 3, approximation algorithms with a finite search horizon may not be able to find a convergent subsequence $\left\{N_{k}(f)\right\}_{k \in \mathbb{N}}$ for every $f \in \mathcal{W}_{\mathrm{T}}$. The question is then for how many functions $f \in \mathcal{X}$ does the algorithm fail?

The non-adaptive method $\left\{\mathrm{T}_{N}\right\}_{N \in \mathbb{N}}$ fails for a residual set in $\mathcal{X}$ whereas Algorithm 1 with an infinite search horizon fails only on a meager set. So we hope that the practical restriction to a finite search horizon does not much increase the divergence set. However, we will see that if an adaptive algorithm with a finite search horizon fails for one $f \in \mathcal{X}$ then it will fail for a whole residual set in $\mathcal{X}$.

To show this, we define the divergence set of all $f \in \mathcal{X}$ for which Algorithm 2 will not be able to find a convergent subsequence.

Definition 2: Let $\mathcal{S}=\left\{S_{k}\right\}_{k \in \mathbb{N}} \subset \mathbb{N}$ be an arbitrary strictly increasing sequence, and denote by $\mathcal{N}(\mathcal{S})$ the set of all $\left\{N_{k}\right\}_{k \in \mathbb{N}} \subset \mathbb{N}$ with $N_{k} \in\left(S_{k}, S_{k+1}\right]$. Then we define

$$
\mathcal{D}(\mathcal{S}):=\left\{f \in \mathcal{X}: \text { for every }\left\{N_{k}\right\}_{k \in \mathbb{N}} \in \mathcal{N}(\mathcal{S}) \text { holds }(6)\right\} .
$$

Theorem 2: If Problem 1 is not solvable then $\mathcal{D}(\mathcal{S})$ is a residual set in $\mathcal{X}$ for every strictly increasing sequence $\mathcal{S}=\left\{S_{k}\right\}_{k \in \mathbb{N}} \subset \mathbb{N}$.

So we have a similar behavior as for non-adaptive methods. If there exists one function such that (6) is satisfied, then there exists a whole residual set of $\mathcal{X}$ for which (6) holds, i.e. for which Algorithm 2 fails. So an adaptive procedure with finite search horizon gives basically no improvement compared to the non-adaptive procedure.

However, we emphasis that there exist weakly divergent approximation sequence $\left\{\mathrm{T}_{N}\right\}_{N}$ for which Algorithm 2 succeeds for all $f \in \mathcal{X}$. So there are situations in which an adaptive method with finite search horizon gives a substantial improvement compared to the non-adaptive method.

Proof: Let $\left\{S_{k}\right\}_{k \in \mathbb{N}}$ be given. For arbitrary $k, M \in \mathbb{N}$, we consider the sets $\mathcal{D}(k ; M)$ which contain all $f \in \mathcal{X}$ such that

$$
\left\|\mathrm{T}_{N} f-\mathrm{T} f\right\|_{\mathcal{Y}}>M \text { for all } N \in\left(S_{k}, S_{k+1}\right] .
$$

It is easy to see that if $\mathcal{D}(k ; M)$ is nonempty for some $k, M \in \mathbb{N}$ then $\mathcal{D}(k ; M)$ is an open set. For all $K, M \in \mathbb{N}$, we define

$$
\overline{\mathcal{D}}(K ; M)=\bigcup_{k=K}^{\infty} \mathcal{D}(k ; M) .
$$

Since $\overline{\mathcal{D}}(K ; M)$ is the union of countable many open sets, it is open. We show that $\overline{\mathcal{D}}(K ; M)$ is dense in $\mathcal{X}$ for all $K, M \in \mathbb{N}$. Let $f \in \mathcal{X}$ and $\epsilon>0$ be arbitrary. Since $\left\{\mathrm{T}_{N}\right\}_{N \in \mathbb{N}}$ converges on a dense subset $\mathcal{X}_{0} \subset \mathcal{X}$, there is $\varphi_{\epsilon} \in \mathcal{X}_{0}$ and $N_{0} \in \mathbb{N}$ such that $\left\|f-\varphi_{\epsilon}\right\|_{\mathcal{X}}<\epsilon$ and such that $\left\|\mathrm{T}_{\mathrm{N}} \varphi_{\epsilon}-\mathrm{T} \varphi_{\epsilon}\right\|_{\mathcal{Y}}<\epsilon / 2$ for all $N \geq N_{0}$. Since Problem 1 is not solvable, there exists an $f_{0} \in \mathcal{X}$ with $\left\|f_{0}\right\|_{\mathcal{X}}=1$ and such that

$$
\limsup _{k \rightarrow \infty} \min _{N \in\left(S_{k}, S_{k+1}\right]}\left\|\mathrm{T}_{N} f_{0}-\mathrm{T} f_{0}\right\|_{\mathcal{Y}}=\infty .
$$

Now we define $f_{\epsilon}:=\varphi_{\epsilon}+\frac{\epsilon}{2} f_{0}$. It is easy to see that $\left\|f-f_{\epsilon}\right\|_{\mathcal{X}}<\epsilon$. Moreover, applying $\left\{T_{N}\right\}_{N \in \mathbb{N}}$ to $f_{\epsilon}$, one obtains

$$
\begin{aligned}
\left\|\mathrm{T}_{N} f_{\epsilon}-\mathrm{T} f_{\epsilon}\right\|_{\mathcal{Y}} & \geq \frac{\epsilon}{2}\left\|\mathrm{~T}_{N} f_{0}-\mathrm{T} f_{0}\right\|_{\mathcal{Y}}-\left\|\mathrm{T}_{N} \varphi_{\epsilon}-\mathrm{T} \varphi_{\epsilon}\right\|_{\mathcal{Y}} \\
& \geq \frac{\epsilon}{2}\left\|\mathrm{~T}_{N} f_{0}-\mathrm{T} f_{0}\right\|_{\mathcal{Y}}-\frac{\epsilon}{2} \quad \text { for all } N \geq N_{0} .
\end{aligned}
$$

Since $f_{0}$ satisfies (9), there exists a $k \geq K$ such that $N_{k} \geq N_{0}$ and such that $\left\|\mathrm{T}_{N} f_{0}-\mathrm{T} f_{0}\right\|_{\mathcal{Y}} \geq 2 M / \epsilon+1$ for some $N \in\left(S_{k}, S_{k+1}\right]$. Consequently, $\left\|\mathrm{T}_{N} f_{\epsilon}-\mathrm{T} f_{\epsilon}\right\|_{\mathcal{Y}} \geq M$ and therefore $f_{\epsilon} \in \overline{\mathcal{D}}(K ; M)$. 
Next, we verify that $\mathcal{D}(\mathcal{S})=\bigcap_{K=1}^{\infty} \bigcap_{M=1}^{\infty} \overline{\mathcal{D}}(K ; M)$. Indeed, assume first $f \in \bigcap_{K=1}^{\infty} \bigcap_{M=1}^{\infty} \overline{\mathcal{D}}(K ; M)$. Then for any arbitrary $K, M \in \mathbb{N}, f \in \overline{\mathcal{D}}(K ; M)$ and there is a $k \geq K$ such that (8) holds, and so $f \in \mathcal{D}(\mathcal{S})$. Conversely, assume $f \in \mathcal{D}(\mathcal{S})$. Then to every $K, M \in \mathbb{N}$ we can find a $k \geq K$ such that (8) is satisfied. Thus $f \in \overline{\mathcal{D}}(K ; M)$ and since $K, M$ can be chosen arbitrary, $f \in$ $\bigcap_{K=1}^{\infty} \bigcap_{M=1}^{\infty} \overline{\mathcal{D}}(K ; M)$.

Each $\overline{\mathcal{D}}(K ; M)$ is open and dense in $\mathcal{X}$, i.e. a residual set. The countable intersection of residual sets is a residual set. Therefore $\mathcal{D}_{1}(\mathcal{N})$ is a residual set in $\mathcal{X}$.

\section{HILBERT TRANSFORM}

This section considers a particular example to illustrate the previous discussions. More details on this example can be found in [12].

For $1 \leq p \leq \infty$, let $L^{p}(\mathbb{T})$ denote the usual set of Lebesgue integrable functions on $\mathbb{T}=[-\pi, \pi)$. For any $f \in L^{1}(\mathbb{T})$, its conjugate function $\widetilde{f}$ is given by the Hilbert transform $\mathrm{H} f$, i.e.

$$
\widetilde{f}(t)=(\mathrm{H} f)(t)=\lim _{\epsilon \rightarrow 0} \frac{1}{2 \pi} \int_{\epsilon \leq|\tau| \leq \pi} \frac{f(t-\tau)}{\tan (\tau / 2)} \mathrm{d} \tau,
$$

where the limit on the right hand side exists for almost all $t \in \mathbb{T}$ (see, e.g., [13, Sect. III.1]). This transformation plays a very important role in many different areas of science and engineering, such as signal processing, communications, control theory, physics $[14,15]$.

We consider the Hilbert transform $\mathrm{H}$ on the Banach space $\mathcal{B}:=\{f \in \mathcal{C}(\mathbb{T}): \widetilde{f}=\mathrm{H} f \in \mathcal{C}(\mathbb{T})\}$ of all $f \in \mathcal{C}(\mathbb{T})$ with a continuous conjugate $\widetilde{f}=\mathrm{H} f$, equipped with the norm $\|f\|_{\mathcal{B}}:=\max \left\{\|f\|_{\infty},\|\mathrm{H} f\|_{\infty}\right\}$. So $\mathcal{B}$ is a proper subset of $\mathcal{C}(\mathbb{T})$. For any $f \in \mathcal{C}(\mathbb{T})$, it conjugate $\widetilde{f}$ belongs to any $L^{p}(\mathbb{T})$ with $p<+\infty$. However, $\tilde{f}$ is not be bounded, in general. In particular, the Hilbert transform of $f \in \mathcal{C}(\mathbb{T})$ is generally not continuous [13].

Hilbert transform from sampled data Our goal is to approximate the Hilbert transform (10) of $f \in \mathcal{B}$ by a sequence $\left\{\mathrm{H}_{N} f\right\}_{N=1}^{\infty}$, where $\mathrm{H}_{N} \in \mathcal{L}(\mathcal{B})$ is assumed to be based on a finite number of samples of $f$. This is a natural requirement in digital signal processing. More precisely, we require that the sequence $\left\{\mathrm{H}_{N}\right\}_{N \in \mathbb{N}} \subset \mathcal{L}(\mathcal{B})$ has the following three properties:

(A) Concentration on a finite sampling set: For every $N \in \mathbb{N}$ there exists a finite set $\Lambda_{N}=\left\{\lambda_{n, N}: n=1, \ldots, M_{N}\right\} \subset \mathbb{T}$ such that for all $f, g \in \mathcal{B}$

$$
\begin{aligned}
& f(\lambda)=g(\lambda) \quad \text { for all } \lambda \in \Lambda_{N} \\
& \text { implies }\left(\mathrm{H}_{N} f\right)(t)=\left(\mathrm{H}_{N} g\right)(t) \quad \text { for all } t \in \mathbb{T} \text {. }
\end{aligned}
$$

(B) Convergence on a dense subset: $\left\{\mathrm{H}_{N}\right\}_{N \in \mathbb{N}} \subset \mathcal{L}(\mathcal{B})$ satisfies $\lim _{N \rightarrow \infty}\left\|\mathrm{H}_{N} f-\widetilde{f}\right\|_{\infty}=0$ for all $f \in \mathcal{C}^{\infty}(\mathbb{T})$, where $\mathcal{C}^{\infty}(\mathbb{T})$ is the subset of infinitely differentiable $f \in \mathcal{C}(\mathbb{T})$.

(C) Generation by a sampling series: There exists a sequence $\left\{\mathrm{A}_{N}\right\}_{N \in \mathbb{N}} \subset \mathcal{L}(\mathcal{B})$ such that $\lim _{N \rightarrow \infty}\left\|\mathrm{A}_{N} f-f\right\|_{\infty}=0$ for all $f \in \mathcal{B}$ and such that $\mathrm{H}_{N} f=\mathrm{HA}_{N} f$ for all $N \in \mathbb{N}$.

Remark: Since the operators $\mathrm{H}_{N}$ are linear, it follows that a sequence $\left\{\mathrm{H}_{N}\right\}_{N \in \mathbb{N}} \subset \mathcal{L}(\mathcal{B})$ has Property (A) if and only if to every $N \in \mathbb{N}$ there exists a finite set $\Lambda_{N}=\left\{\lambda_{1, N}, \ldots, \lambda_{M_{N}, N}\right\} \subset \mathbb{T}$ and functions $\left\{h_{1, N}, \ldots, h_{M_{n}, N}\right\} \subset \mathcal{B}$ such that

$$
\mathrm{H}_{N} f=\sum_{n=1}^{M_{N}} f\left(\lambda_{n, N}\right) h_{n, N} \quad \text { for all } f \in \mathcal{B} .
$$

It was shown in [2] that every sequence $\left\{\mathrm{H}_{N}\right\}_{N \in \mathbb{N}} \in \mathcal{L}(\mathcal{B})$ with properties (A), (B), (C) shows a divergence behavior as in (1) on $\mathcal{B}$. It was conjectured later that any sequence $\left\{\mathrm{H}_{N}\right\}_{N \in \mathbb{N}}$ with properties (A), (B), (C) is also strongly divergent [16]. The interesting question here is whether we can apply the adaptive Algorithm 2 to find convergent subsequences $\left\{N_{k}(f)\right\}_{k \in \mathbb{N}}$ such that $\mathrm{T}_{N_{k}} f$ converges to $\mathrm{T} f$. Since we are interested in methods which can be implemented in practice, we ask for methods with a finite search horizon.

The following theorem shows that the answer is actually negative, i.e. there exists no sampling based adaptive approximation method for the Hilbert transform which can be realized in practice.

Theorem 3: Let $\left\{\mathrm{H}_{N}\right\}_{N \in \mathbb{N}} \subset \mathcal{L}(\mathcal{B})$ be a sequence with properties (A)-(C), and let $\left\{S_{k}\right\}_{k \in \mathbb{N}} \subset \mathbb{N}$ be an arbitrary strictly increasing sequences. There is a function $f_{*} \in \mathcal{B}$ such that

$$
\limsup _{k \rightarrow \infty} \min _{N \in\left(S_{k}, S_{k+1}\right]}\left\|\mathrm{H}_{N} f_{*}\right\|_{\infty}=\infty .
$$

Sketch of proof: We apply a similar construction as in the proof of [16, Theorem 7]. There we constructed $f_{*} \in \mathcal{B}$ and two strictly increasing sequences $\left\{N_{r}^{(1)}\right\}_{r \in \mathbb{N}},\left\{N_{r}^{(2)}\right\}_{r \in \mathbb{N}} \subset \mathbb{N}$ with $N_{r}^{(2)}>$ $N_{r}^{(1)}$ and

$$
\left\|\mathrm{H}_{N} f_{*}\right\|_{\infty} \geq r-C_{0} \quad \text { for all } N \in\left[N_{r}^{(1)}, N_{r}^{(2)}\right]
$$

for all $r \in \mathbb{N}$ and with a certain constant $C_{0}>0$. Here we choose the elements of the sequences $\left\{N_{r}^{(1)}\right\}_{r \in \mathbb{N}}$ and $\left\{N_{r}^{(2)}\right\}_{r \in \mathbb{N}}$ from the given set $\left\{S_{k}\right\}_{k \in \mathbb{N}}$ and not from $\mathbb{N}$ as in [16]. Then we define

$$
\widehat{N}_{2 r-1}:=N_{r}^{(1)} \quad \text { and } \quad \widehat{N}_{2 r}:=N_{r}^{(2)} \quad \text { for all } r=1,2,3, \ldots
$$

This yields a strictly increasing subsequence $\left\{\widehat{N}_{r}\right\}_{r \in \mathbb{N}}$ of $\left\{S_{k}\right\}_{k \in \mathbb{N}}$ and by the construction of $f_{*} \in \mathcal{B}$, we have $\left\|\mathrm{H}_{N} f_{*}\right\|_{\infty} \geq r-C_{0}$ for all $N \in\left[\widehat{N}_{r}, \widehat{N}_{r+1}\right]$ and for any $r \in \mathbb{N}$. Consequently

$$
\lim _{r \rightarrow \infty} \min _{N \in\left[\widehat{N}_{r}, \widehat{N}_{r+1}\right]}\left\|\mathrm{H}_{N} f_{*}\right\|_{\infty}=\infty .
$$

Since $\left\{\widehat{N}_{r}\right\}_{r \in \mathbb{N}}$ is a subsequence of $\left\{S_{k}\right\}_{k \in \mathbb{N}}$, it is clear that to every $r \in \mathbb{N}$ there exists a $k(r) \in \mathbb{N}$ such that $\left(S_{k(r)}, S_{k(r)+1}\right] \subset$ $\left[\widehat{N}_{r}, \widehat{N}_{r+1}\right]$, and that

$$
\min _{N \in\left[S_{k(r)}, S_{k(r)+1}\right]}\left\|\mathrm{H}_{N} f_{*}\right\|_{\infty} \geq \min _{N \in\left(\widehat{N}_{r}, \widehat{N}_{r+1}\right]}\left\|\mathrm{H}_{N} f_{*}\right\|_{\infty}
$$

for all $r \in \mathbb{N}$. Together with (11), this proves the theorem.

Theorem 3 shows that there are functions $f \in \mathcal{B}$ such that the norm $\left\|\mathrm{H}_{N} f\right\|_{\infty}$ of any sampling based linear approximation $\mathrm{H}_{N} f$ exceeds any given bound for any given number of sufficiently large consecutive approximation indices $N$. So no matter how large we choose the length of the search intervals $\left(S_{k}, S_{k+1}\right]$ in Algorithm 2, there always exist functions $f \in \mathcal{B}$ for which the algorithm fails. Together with Theorem 2 we obtain the following statement.

Corollary 4: Let $\left\{\mathrm{H}_{N}\right\}_{N \in \mathbb{N}} \subset \mathcal{L}(\mathcal{B})$ be a weakly divergent approximation sequence of $\mathrm{H}$ with properties $(A),(B),(C)$. Then Problem 1 is not solvable and the corresponding divergence set $\mathcal{D}(\mathcal{S})$ is residual in $\mathcal{B}$ for every strictly increasing sequence $\mathcal{S}=\left\{S_{k}\right\}_{k \in \mathbb{N}}$.

So Corollary 4 shows that there exist no sampling based adaptive approximation method with finite search horizon for the Hilbert transform. Nevertheless, we note that if we allow for an infinite search horizon, then there exist adaptive methods which convergence for all function in a residual subset of $\mathcal{B}$. 


\section{REFERENCES}

[1] M. Unser, A. Aldroubi, and M. Eden, "B-spline signal processing. Part I \& II," IEEE Trans. Signal Process., vol. 41, no. 2, pp. 821-848, Feb. 1993.

[2] H. Boche and V. Pohl, "On the calculation of the Hilbert transform from interpolated data," IEEE Trans. Inf. Theory, vol. 54, no. 5, pp. 2358-2366, May 2008.

[3] S. Banach and H. Steinhaus, "Sur le principle de la condensation de singularites," Fund. Math., vol. 9, pp. 50-61, 1927.

[4] S. Banach, "Über die Baire'sche Kategorie gewisser Funktionenmengen," Studia Math., vol. 3, no. 1, pp. 174-179, 1931.

[5] L. V. Kantorovich and G. P. Akilov, Functional analysis in normed spaces. New York: Pergamon Press, 1964.

[6] H. Boche and V. Pohl, "System approximations and generalized measurements in modern sampling theory," in Sampling Theory, a Renaissance, ser. Applied and Numerical Harmonic Analysis, G. Pfander, Ed. New York: Springer-Birkhäuser, 2015, pp. 269-305.

[7] H. Boche and B. Farrell, "Strong divergence of reconstruction procedures for the Paley-Wiener space $\mathcal{P} \mathcal{W}_{\pi}^{1}$ and the Hardy space $\mathcal{H}^{1}$, , J. Approx. Theory, vol. 183, pp. 98-117, Jul. 2014.

[8] A. Zygmund, Trigonometric Series, 3rd ed. Cambridge, UK: Cambridge University Press, 2002.
[9] L. Fejér, "A Fourier-féle sorokől," in Leopold Fejér Gesammelte Arbeiten, P. Turán, Ed. Basel: Birkhäuser, 1970, vol. 1, pp. 264-297.

[10] H. Boche and U. J. Mönich, "Strong divergence for system approximations," Probl. Inf. Transm., vol. 51, no. 3, pp. 240266, 2015.

[11] J. C. Oxtoby, Measure and Category, 2nd ed. New York: Springer-Verlag, 1980.

[12] H. Boche and V. Pohl, "On the strong divergence of Hilbert transform approximations and a problem of Ul'yanov," J. Approx. Theory, to appear, 2016 (DOI: 10.1016/j.jat.2016.01.002).

[13] J. B. Garnett, Bounded Analytic Functions. New York: Academic Press, 1981.

[14] F. W. King, Hilbert Transforms, ser. Encyclopedia of Mathematics and its Applications. Cambridge: Cambridge University Press, 2009.

[15] V. Pohl and H. Boche, Advanced Topics in System and Signal Theory: A Mathematical Approach, ser. Foundations in Signal Processing, Communications and Networking. Berlin: Springer-Verlag, 2009.

[16] H. Boche and V. Pohl, "On the strong divergence of Hilbert transform approximations from sampled data," in Proc. 11th Intern. Conf. on Sampling Theory and Applications (SampTA), Washington, DC, USA, May 2015, pp. 216-220. 